\title{
First Report of Bacteremia Caused by Clostridium cadaveris in China
}

\author{
Xiangyun $\mathrm{Li}^{\prime}$ \\ Xiujie $\mathrm{Wu}^{2}$ \\ Yuanhong $\mathrm{Xu}$ (D) ${ }^{1, *}$ \\ Yajing Liu ${ }^{3-5, *}$ \\ 'Department of Laboratory Medicine, \\ The First Affiliated Hospital of Anhui \\ Medical University, Hefei, Anhui, People's \\ Republic of China; ${ }^{2}$ Department of \\ Laboratory Medicine, Anhui Medical \\ University, Hefei, Anhui, People's \\ Republic of China; ${ }^{3}$ Department of \\ Obstetrics and Gynecology, The First \\ Affiliated Hospital of Anhui Medical \\ University, Hefei, Anhui, People's \\ Republic of China; ${ }^{4} \mathrm{NHC}$ Key Laboratory \\ of Study on Abnormal Gametes and \\ Reproductive Tract, Anhui Medical \\ University, Hefei, Anhui, People's \\ Republic of China; ${ }^{5}$ Anhui Province Key \\ Laboratory of Reproductive Health and \\ Genetics, Anhui Medical University, \\ Hefei, Anhui, People's Republic of China \\ *These authors contributed equally to \\ this work
}

Background: Bacteremia caused by $C$. cadaveris is an extremely rare infection that accounts for $0.5-2 \%$ of all positive blood cultures. C. cadaveris is an opportunistic agent that is highly lethal in immunocompromised or epithelial barrier disruption hosts.

Case Presentation: A 74-year-old woman was admitted to our hospital with a high fever. The patient was diagnosed with an abdominal infection after a bowel invasion and recurrence of an ovarian tumor after surgery. Blood sample culturing yielded transparent, smooth, moist, slightly raised colonies without a hemolytic ring. C. cadaveris was identified by gram staining and MALDI-TOF MS. 16S rRNA gene sequencing confirmed a sequence homologous to C. cadaveris in the GenBank database. Drug susceptibility testing showed that $C$. cadaveris was sensitive to cefoxitin, clindamycin, imipenem, meropenem, metronidazole, and vancomycin. After treatment, the patient's body temperature was normal and she was discharged from the hospital.

Conclusion: This is the first case report of bacteremia caused by C. cadaveris in China and also the first report of identifying $C$. cadaveris in clinical specimens by MALDI-TOF MS. This case study could increase the awareness of these infections in China.

Keywords: C. cadaveris, bacteremia, rapid identification, drug sensitivity test

\section{Introduction}

Clostridium cadaveris ( $C$. cadaveris) is a motile, anaerobic, gram-positive straight rod bacterium that is most prominent during the decay of dead bodies. It was first reported by Klein in $1899 .{ }^{1,2}$ C. cadaveris is widely distributed in the environment and can be isolated from soil, marine sediment, and cobra venom. ${ }^{3,4}$ It has been found in intestinal tracts of humans and is excreted in feces. ${ }^{5,6}$ Pathologically, C. cadaveris produces toxins and is occasionally isolated from abscesses and wound infections, with extremely rare episodes of bacteremia resulting in positive blood cultures. Only a few reports of bacteremia have been reported, for example, Knight et al reported a case in Australia and Schade et al reported two cases in the Netherlands. ${ }^{7,8,15}$ In this article, we provide the first report of a bacteremia case caused by $C$. cadaveris in China. We analyzed the first C. cadaveris clinical isolate by matrix-assisted laser desorption ionization-time-of-flight mass spectrometry (MALDI-TOF MS) and summarized the rapid identification and results of antimicrobial susceptibility testing.

\section{Materials and Methods}

\section{Blood Cultures and Isolates Identification of $C$. cadaveris}

One strain of $C$. cadaveris was isolated from a positive blood anaerobic culture in the Department of Laboratory Medicine at the First Affiliated Hospital of Anhui
Correspondence: Yajing Liu; Yuanhong Xu Email liuyajing627I@163.com; xyhong1964@163.com 
Medical University in 2020. One drop of blood was inoculated onto Columbia blood plate medium, and the anaerobic package was added at $37^{\circ} \mathrm{C}$ and in a $5 \% \mathrm{CO}_{2}$ chamber. The colonies were taken for gram staining and microscopic examination for primary identification.

\section{MALDI-TOF MS Identification}

MALDI-TOF MS was performed using a Vitek MS platform by the direct smear method according to the instructions of the manufacturer. After the acquisition of the spectra, data were transferred to the analysis server, which used software algorithms to compare the generated spectrum with the typical spectra within the database.

\section{I6S rRNA Gene Sequencing Identification and Construction of a Phylogeny Tree}

The original bacteria were purified and expanded to extract genomic DNA. A forward primer $27 \mathrm{~F}$ and a reverse primer 1492R were used for PCR amplification. The sequencing was compared with the $16 \mathrm{~S}$ rRNA gene sequencing of known bacteria in the GenBank database. The phylogenetic tree was established using the MEGA7.0 software.

\section{In vitro Drug Susceptibility Testing}

All tests were performed using the agar dilution method.

\section{Case Report}

A 74-year-old woman was admitted to our hospital with a high fever due to recurrence of an ovarian tumor following surgery. About 2 years ago, the patient had resections of the whole uterus, bilateral adnexa, greater omentum, and appendix. Postoperative pathological examinations revealed the presence of poorly differentiated adenocarcinoma with necrosis in the ovaries and cancer cells were found in the greater omentum and appendix. The patient was treated with chemotherapy. No obvious bone marrow suppression was observed following treatment, and periodic reviews were conducted. One year later, the patient had a CA125 level of $66.04 \mathrm{U} / \mathrm{mL}$ and human epididymis secreted proteins 4 (HE4) level of 209.70pmol/L. CT examination suggested postoperative recurrence of ovarian cancer, and multiple cystic foci were observed in the right upper abdomen, spleen, and perispleen. Multiple metastases were considered.

During hospitalization, the patient had a fever with an axillary temperature reaching $39.3^{\circ} \mathrm{C}$. On laboratory investigations, the patient had a procalcitonin (PCT) level of
$0.57 \mathrm{ng} / \mathrm{mL}$, a CRP level of $148.42 \mathrm{mg} / \mathrm{L}$, a WBC count of $3.76 \times 10^{9} / \mathrm{L}$, and a neutrophil percentage of $80.4 \%$. Blood culture was conducted in aerobic and anaerobic flasks. The positive bacteria were identified as $C$. cadaveris in an anaerobic flask after $16.4 \mathrm{~h}$. Considering abdominal infection due to bowel invasion caused by $C$. cadaveris, the patient was treated with imipenem and cilastatin sodium (1 g, every $8 \mathrm{~h}$, iv. drip) for anti-infection and hydrocortisone sodium succinate $(0.01 \mathrm{~g}$, once, iv. drip) for antiinflammation. After 2 days, the patient's body temperature was normal and she was discharged from hospital.

\section{Results}

After culturing for $48 \mathrm{~h}$, gray, translucent, smooth, slightly raised colonies with a diameter of $0.5-1 \mathrm{~mm}$ were visible without a hemolytic ring (Figure 1). The gram staining results showed a positive bacillus (Figure 2). The MALDI-TOF MS of the isolated strain was most homologous to $C$. cadaveris with a confidence level of $99.9 \%$ (Figure 3 ). The PCR product was identical to the $16 \mathrm{~S}$ rRNA gene sequencing of known C. cadaveris. By constructing a phylogenetic tree, it is found that that isolate was located in the same branch as $C$. cadaveris (Figure 4). The isolate was confirmed, and the entry number is NR 104695.1. The strain was sensitive to cefoxitin, clindamycin, imipenem, meropenem, metronidazole, and vancomycin. The minimum inhibitory concentration (MIC) is shown in Table 1. The MIC was defined as the concentration of the drug that completely inhibited growth or caused a marked

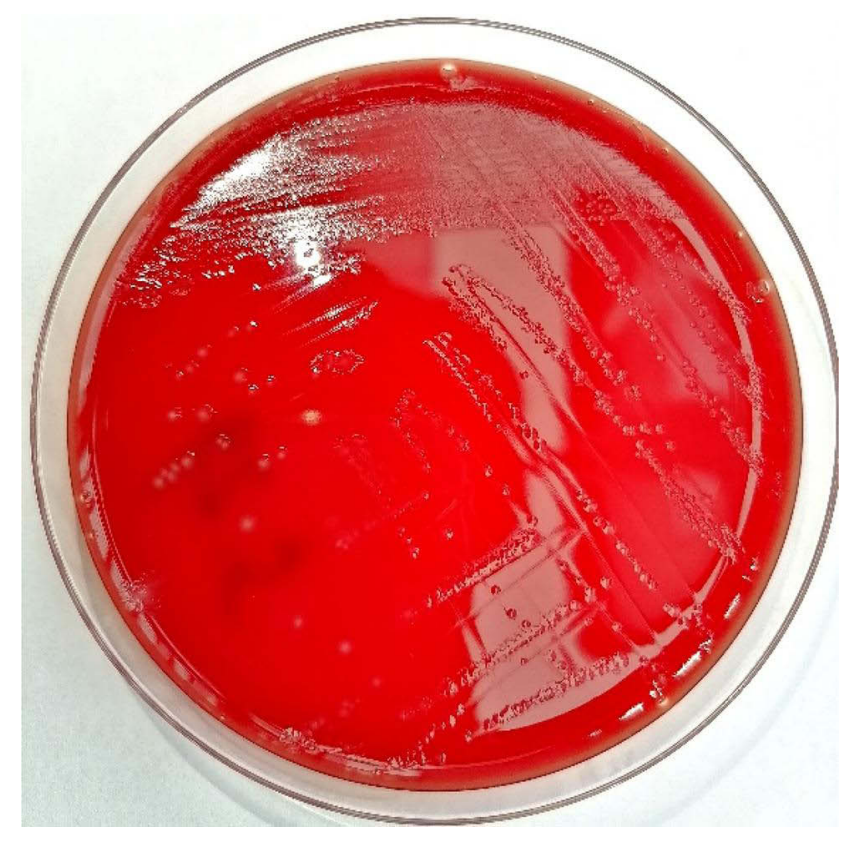

Figure I Result of pure culture of the bacterial isolate after $48 \mathrm{~h}$. 


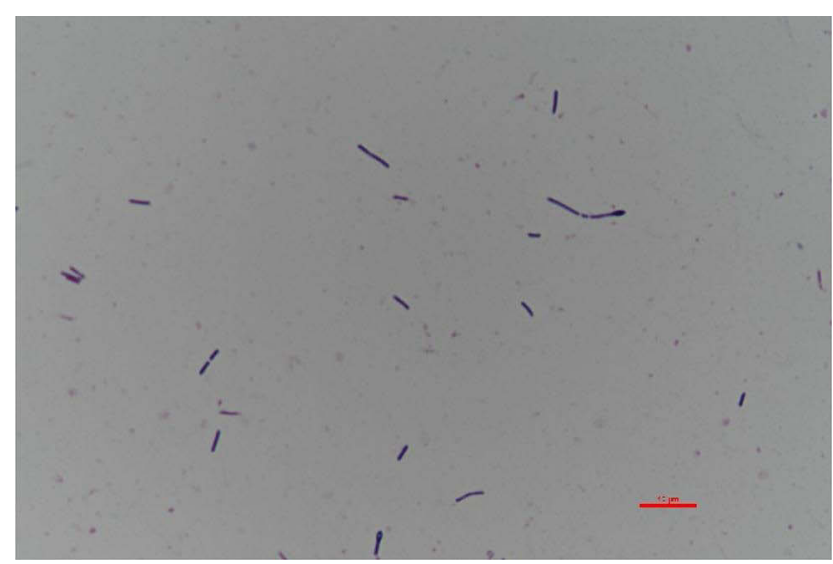

Figure 2 Gram staining of the bacterial isolate.

reduction $(50 \%$ or $90 \%)$ in the presence of growth compared to the drug-free growth control.

\section{Discussion}

Accurate identification of obligate anaerobic, gram-positive bacilli is difficult as they are small, fastidious, and biochemically inert. In the past, it has been particularly challenging to isolate $C$. cadaveris from clinical samples. Recently, MALDI-TOF MS has become widely established in clinical microbiology laboratories as an effective method to identify bacteria and fungi ${ }^{12,13}$ with an accuracy of $90 \%$ or higher. In this report, MALDI-TOF MS was used to identify an isolated strain of $C$. cadaveris with a confidence of $99.9 \%$. This is the first report of $C$. cadaveris in China and also the first report of identifying $C$. cadaveris in clinical specimens by MALDI-TOF MS technology. 16S rRNA gene sequencing and MALDI-TOF MS were both performed on the strain. A phylogenetic tree was constructed to confirm the correctness of the pathogen. The results of 16S rRNA gene sequencing and MALDI-TOF MS were consistent, and both achieved high accuracy.

C. cadaveris is an opportunistic pathogen that causes infection problems in immunocompromised patients. ${ }^{9,10}$ C. cadaveris infection is usually associated with a poor physical condition, underlying malignancy, and severe immune suppression. C. cadaveris has been found in the human intestinal tract and is the source of infection in most abdominal bacteremia cases. ${ }^{11}$ In this report, the patient had recurrent ovarian cancer and intestinal invasion resulting in abdominal infection and bacteremia. Knight et al reported a case of a 19-year-old man who had C. cadaveris bacteremia with associated superior mesenteric vein thrombus. Following treatment with intravenous metronidazole, piperacillin/tazobactam, and oral vancomycin, and the patient demonstrated a gradual clinical improvement and resolution of symptoms. ${ }^{8}$ Citron et al evaluated the in vitro activity of glycolipodepsipeptide antibiotics (Ramoplanin, Teicoplanin, and Vancomycin)

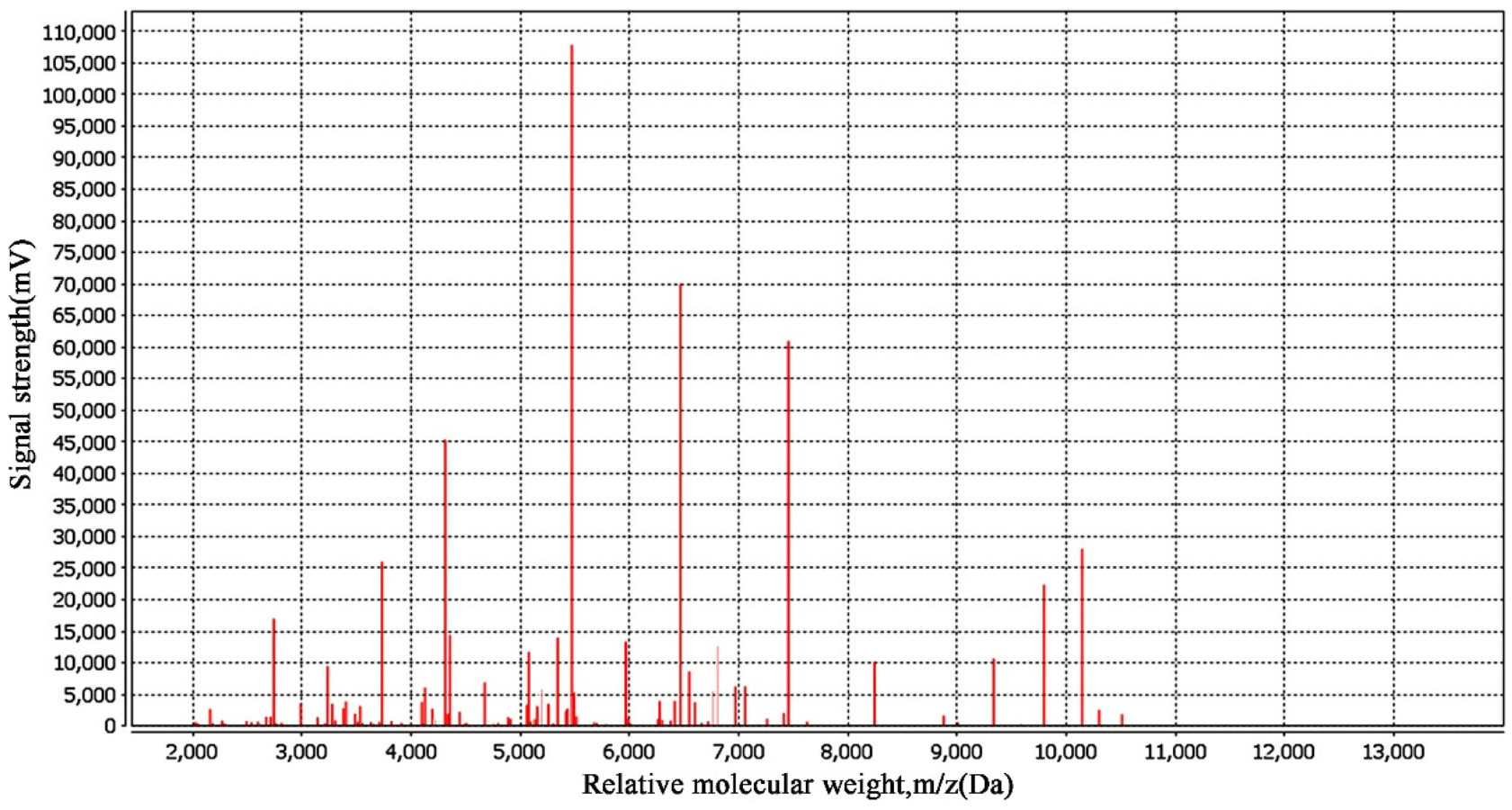

Figure 3 Mass spectra of the bacterial isolate. 


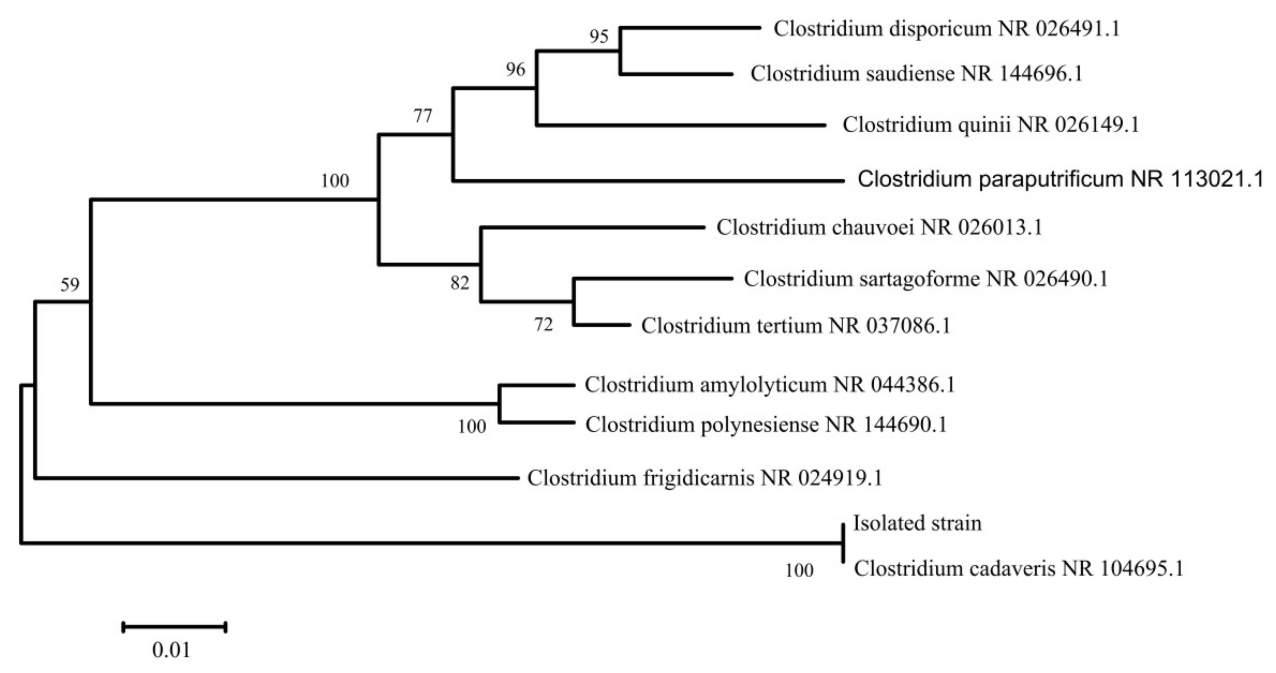

Figure 4 Phylogenetic tree of the bacterial isolate.

using an agar dilution method to identify drugs that were active against $C$. cadaveris. ${ }^{14}$ In this report, susceptibility testing was performed according to the reference agar dilution method and the MIC results of cefoxitin, clindamycin, imipenem, meropenem, metronidazole, and vancomycin were consistent with Goldstein's results. The drugs exhibited potent activity against $C$. cadaveris. In the case of our patient, imipenem and cilastatin sodium were chosen as antibiotics and the patient showed a strong clinical response.

\section{Conclusions}

In this report, one strain of $C$. cadaveris was isolated and identified by MALDI-TOF MS and 16S rRNA gene sequencing as a rapid and accurate detection method that was performed within $24 \mathrm{~h}$. After treatment with imipenem and cilastatin sodium, the patient's body temperature returned to normal. Accurate diagnosis and selection of effective treatments are of particular importance in cases involving rare bacteria.

Table I Drug Sensitivity Results of Bacterial Isolate

\begin{tabular}{|l|l|l|l|}
\hline Antibiotics & MIC (mg/L) & $\mathbf{5 0 \%}$ & $\mathbf{9 0 \%}$ \\
\hline Cefoxitin & 2 & 0.5 & $\mathrm{I}$ \\
Clindamycin & 2 & 0.5 & $\mathrm{I}$ \\
Imipenem & 4 & $\mathrm{I}$ & 2 \\
Meropenem & 4 & $\mathrm{I}$ & 2 \\
Metronidazole & 0.5 & 0.125 & 0.25 \\
Vancomycin & 4 & $\mathrm{I}$ & 2 \\
\hline
\end{tabular}

\section{Acknowledgments}

This work was supported by the Opening Project of Anhui Province Key Laboratory of Reproductive Health and Genetics, Youth Project of National Natural Science Foundation of China (82100613), Doctoral Research Foundation of the First Affiliated Hospital of Anhui Medical University (Bsky2019038), Scientific Research Fund of Anhui Medical University (2021xkj137), and Anhui Provincial Key Research and Development Plan Project (201904a07020049). The patient's legal guardian signed the written informed consent regarding publication of their respective photograph and case detail in a journal article. All clinical data of the patients were collected in accordance with the Local Research Ethics committee of the First Affiliated Hospital of Anhui Medical University (Quick-PJ2021-13-33).

\section{Disclosure}

The authors have no conflicts of interest to declare.

\section{References}

1. Klein E. [Ein beitrag zur bakteriologie der leichenverwesung]. Zentralbl Bakteriol Parasitenkd Infektionskr Hyg. [A contribution to the bacteriology of corpse decay]. 1899;1:278-284. German.

2. Schade R, Van Rijn M, Timmers H, et al. Clostridium cadaveris bacteraemia: two cases and review. Scand $J$ Infect Dis. 2005;38:59-78. doi:10.1080/00365540500388792

3. Willis AT. Characteristics of pathogenic and related clostridia. In: Willis AT, editor. Anaerobic Bacteriology: Clinical and Laboratory Practice. Boston: Butterworth; 1977:111-166.

4. Pfaff CE, Droege CA, Magner ME, et al. Clostridium cadaveris bacteraemia in a critically ill surgical patient with septic shock after total abdominal colectomy: a case report and review of literature. Glob Druag Ther. 2016;2:1-4. doi:10.15761/GDT.1000111 
5. Kiu R, Caim S, Alcon-Giner C, et al. Preterm infant-associated clostridium tertium, Clostridium cadaveris, and clostridium paraputrificum strains: genomic and evolutionary insights. Genome Biol Evol. 2017;9(10):2707-2714. doi:10.1093/gbe/evx210

6. Shinha T. Bacteraemia due to Clostridium cadaveris. Infect Dis Clin Pract. 2016;24:232-233. doi:10.1097/IPC.0000000000000274

7. Poduval RD, Mohandas R, Unnikrishnan D, et al. Clostridium cadaveris bacteremia in an immunocompetent host. Clin Infect Dis. 1999;29(5):1354-1355. doi:10.1086/313491

8. Knight CG, Heitmann PT, McDonald CR. Clostridium cadaveris bacteraemia with associated superior mesenteric vein thrombus. ANZ J Surg. 2021;91(7-8):E531-E532. Epub 2021 Jan 8. doi:10.1111/ans. 16538

9. Corrigan RA, Lomas-Cabeza J 1, Stubbs D 1, McNally M. Clostridium cadaveris osteomyelitis: an unusual pathogen which highlights the importance of deep tissue sampling in chronic osteomyelitis. J Bone Jt Infect. 2020;35(2):96-100. doi:10.7150/ jbji.43801.eCollection2020

10. Gucalp R, Motyl M, Carlisle P, et al. Clostridium cadaveris bacteraemia in the immunocompromised host. Med Pediatr Oncol. 1993;21 (6):70-72. doi:10.1002/mpo.2950210114
11. Yan J, Hinds R, Burgner D. Clostridium cadaveris splenic abscess in an adolescent. J Paediatr Child Health. 2018;54(4):460-461. doi:10.1111/jpc.13893

12. Morshed S, Malek F, Silverstein RM, et al. Clostridium cadaveris septic arthritis after total hip arthroplasty in a metastatic breast cancer patient. $J$ Arthroplasty. 2007;22(2):289-292. doi:10.1016/j. arth.2006.02.158

13. Koo T, Lee J, Hwang S. Development of an interspecies interaction model: an experiment on Clostridium cadaveris and Clostridium sporogenes under anaerobic condition. J Environ Manage. 2019;237 (1):247-254. doi:10.1016/j.jenvman.2019.02.084

14. Citron DM, Merriam CV, Tyrrell KL, et al. In vitro activities of ramoplanin, teicoplanin, vancomycin, linezolid, bacitracin, and 4 other antimicrobials against intestinal anaerobic bacteria. Anti Agents Chemother. 2003;47(7):2334-2338. doi:10.1128/AAC.47.7.2 334-2338.2003

15. Schade RP, Van Rijn M, Timmers HJLM, et al. Clostridium cadaveris bacteraemia: two cases and review. Scand J Infect Dis. 2006;38 (1):59-62. doi:10.1080/00365540500388792
Infection and Drug Resistance

\section{Publish your work in this journal}

Infection and Drug Resistance is an international, peer-reviewed openaccess journal that focuses on the optimal treatment of infection (bacterial, fungal and viral) and the development and institution of preventive strategies to minimize the development and spread of resistance. The journal is specifically concerned with the epidemiology of
Dovepress

antibiotic resistance and the mechanisms of resistance development and diffusion in both hospitals and the community. The manuscript management system is completely online and includes a very quick and fair peerreview system, which is all easy to use. Visit http://www.dovepress.com/ testimonials.php to read real quotes from published authors. 